\section{Case Reports in Oncology}

\title{
A Rare Differential Diagnosis of an Adrenal Mass: A Case Report
}

\author{
Ann-Katrin Seidel ${ }^{a} \quad$ Miklos Pless $^{a} \quad$ Christian Michel $^{b} \quad$ Christopher Soll $^{c}$ \\ Caroline Hochuli $^{d}$ Jacques Gubler ${ }^{\mathrm{e}}$ \\ aDepartment of Medical Oncology, Kantonsspital Winterthur, Winterthur, Switzerland; \\ ${ }^{b}$ Eberhard Karls University of Tübingen, Tübingen, Germany; ${ }^{c}$ Department of Visceral und \\ Thoracic Surgery, Kantonsspital Winterthur, Winterthur, Switzerland; dDepartment of \\ Medicine, Spital Lachen, Lachen, Switzerland; 'Department of Infectious Diseases, \\ Kantonsspital Winterthur, Winterthur, Switzerland
}

\section{Keywords}

Alveolar echinococcosis of the adrenal gland · Mimicking cancer · Second cancer

\section{Abstract}

Ten years after his last treatment for diffuse large B-cell lymphoma, a seemingly healthy, 64year-old man presented for his lymphoma-related follow-up. Ultrasound revealed an impressive tumor in the right adrenal gland. Due to recurrent cancer therapies in the past, this seemed highly suspicious of a second malignancy, such as primary adrenal carcinoma. Surprisingly, histology disclosed a very different but rare cause for this pseudotumorous lesion.

(C) 2017 The Author(s)

Published by S. Karger AG, Basel

\section{Introduction}

One of the most serious late sequelae of cancer therapy in long-term survivors is the development of second cancers [1]. The risk after initial cancer therapy is increased for up to 15 years [2], while the frequency and type of second cancers vary, depending on treatment, genetic predisposition, and lifestyle factors [3]. 
Life expectancy of patients with aggressive non-Hodgkin lymphoma (NHL) has substantially increased since the introduction of novel therapies [4]. Accordingly, NHL survivors are at increased risk for second cancers, such as myelodysplastic syndrome, acute myelocytic leukemia, and several solid tumors. Alkylating chemotherapy, which is substantial in therapy of aggressive lymphomas, has especially been linked to the development of several types of second cancers [1].

Accordingly, the detection of second cancers in NHL survivors is of growing importance regarding adequate follow-up care, besides identifying a relapse and monitoring nonneoplastic long-term side effects [2]. Current NICE Guidelines reflect this in a long-term follow-up plan for health care professionals [5].

In the present case, this follow-up procedure confronted us with a particular diagnostic challenge.

\section{Case Report}

In January 2016, a 64-year-old asymptomatic male patient presented for his lymphomarelated annual follow-up after he had been treated repeatedly for relapsing diffuse large Bcell lymphoma (DLBCL) between 1996 and 2006. His initial diagnosis of DLBCL in 1996 was made after resection of a stenosing tumor in the ileocecal region. Consecutively, he underwent adjuvant chemotherapy with CHOP (cyclophosphamide, hydroxydoxorubicin, vincristine, prednisone), and remained in remission until 2002. In 2002, he developed a first relapse in a single cervical lymph node, which was successfully treated with curative radiotherapy ( 40 Gy cumulative dose to the neck and supraclavicular region). A second relapse in both tonsils and inguinal lymph nodes appeared in 2005 and was treated by salvage chemotherapy with R-EPOCH (rituximab, etoposide, prednisone, vincristine, cyclophosphamide, doxorubicin) and subsequent high-dose chemotherapy (cyclophosphamide, carmustine, etoposide) followed by an autologous stem cell retransfusion. Since then, he has been in ongoing third complete remission.

Annual follow-up with ultrasound in 2016 revealed a large tumor of $7 \times 7 \mathrm{~cm}$ in the right adrenal gland. FDG-PET (fluorodeoxyglucose-positron emission tomography) confirmed an FDG active lesion in the right adrenal gland (SUV 8.3) (Fig. 1) and two additional small subpleural lesions of 5 and $19 \mathrm{~mm}$ (SUV max. 3.1), located in the lateral segment of the middle lobe.

Based on imagery results, our initial assumption was either primary adrenal carcinoma or metastatic lung cancer, while a relapsed DLBCL was unlikely, due to absence of lymphadenopathy.

Surprisingly, diagnostic biopsy of the adrenal mass and the pulmonary lesions did not reveal any malignant cells, but a granulomatous inflammatory reaction with PAS (periodic acid-Schiff)-positive membranes consistent with parasitic membranes. Particularly, the specimen showed evidence of calcareous corpuscles and small hooks, suspicious of Echinococcus multilocularis. The positive serology with detectable monoclonal antibody Em2G11 and Em18 in immunohistochemistry confirmed the diagnosis of alveolar echinococcosis (AE).

MRI of the upper abdomen detected 3 hepatic lesions, 1 small lesion in segment 7 with infiltrative growth into the right adrenal gland (Fig. 2), and 2 further satellite lesions in segments 6 and 8 . 
Treatment with albendazole $(2 \times 400 \mathrm{mg} /$ day $)$ was administered. In a 2 -step procedure, the parasitic lesions were surgically resected, necessitating en bloc adrenalectomy, hemihepatectomy, and resection of the middle lobe of the lung (Fig. 3). Parasite-free resection margins (R0 resection) were obtained. Postoperatively, the dose of albendazole had to be reduced due to hepatic toxicity. Latest FDG-PET-CT imaging showed no evidence for recurrence 6 months after the operation. Retrospective analysis of previous CT scans revealed the presence of a very small hepatic lesion already 10 years prior to the present manifestation.

\section{Discussion}

Cancer survivors are at an increased risk of developing second cancer after treatment with radiotherapy and chemotherapy, even for up to 15 years after initial treatment $[2,6]$. Alkylating substances like chlorambucil, ifosfamid, cyclophosphamide, carmustine, lomustine, melphalan, and topoisomerase inhibitors like etoposide are especially linked to the development of second cancers [1]. A US study with 77,823 patients detected an increased relative risk of 1.15 for patients with NHL, while a British study detected an even higher relative risk of 1.4 for DLBCL survivors [6, 7]. In particular, elevated incidences for second cancers have been reported for myelodysplastic syndrome, acute myelocytic leukemia, and several solid tumors like cancer of the lung, bladder, stomach, head and neck, thyroid, skin, mesothelioma and Hodgkin's lymphoma $[2,6,8]$.

Our patient was at increased risk of developing a second cancer due to radiation therapy (40 Gy) and various cytotoxic therapies, most notably with cyclophosphamide, carmustine, and etoposide. Two late relapses and the history of intense treatment justified the long-term follow-up with routine ultrasound as late as 10 years after the third complete remission.

Although imaging findings in the adrenal gland and lungs were highly suspicious of second cancer, we ruled out malignancy histologically.

One remaining, although improbable, differential diagnosis for adrenal tumor was adrenal $\mathrm{AE}$, a parasitic infection caused by the tapeworm E. multilocularis. The parasite E. multilocularis is prevalent in the northern latitudes of Central Europe as well as in Asia and North America. In Germany, about 40 new infections per year occur, while the estimations for Switzerland are between 10 and 20 new infections per year $[9,10]$. Infection occurs when eggs of E. multilocularis, usually excreted by foxes, are accidentally ingested by humans. In $99 \%$ of all cases, AE is initially located in the liver and spreads into other organs by infiltration or metastasis formation. Such multiorgan disease is described in about $13 \%$ of all cases, usually with involvement of the lungs, spleen, or brain [11]. Adrenal AE, however, is rare, while primary adrenal AE is even rarer with only about 7 cases reported in the literature so far [12-14].

The first choice of therapy for AE would be radical resection in all cases suitable for total resection [11]. Additional therapy with benzimidazoles (e.g., mebendazole, albendazole) is required for all patients for at least 2 years after complete resection, and for life in all other cases [11].

The decision for the adequate treatment in this case was compounded by the fact that ultrasound could not fully rule out a potential infiltration of the caval vein, which could have created significant risk and necessitated greater surgical effort. Nevertheless, the patient decided in favor of surgery, the only curative treatment option. Although the intervention was challenging, the surgeon successfully removed the coating Echinococcus covering the caval vein and all infected tissue. 
However, how could echinococcosis be mistaken for second cancer in this patient? AE generally shows characteristic morphologic features in ultrasound, CT, and MRI; however, in some rare cases, both morphology and anatomic location can mimic malignant cancer [15]. In our case, the major lesion appeared to be in the adrenal gland, which is an extremely rare primary lesion for AE. Only additional high-resolution liver MRI was able to detect a tiny primary lesion in liver segment 7 with continuous growth into the right adrenal gland.

\section{Conclusion}

AE may mimic malignancy both in clinical presentation and radiological imaging. Accordingly, even in seemingly clear cases, vigilance is required for alternative diagnoses. Although echinococcosis is rare, the incidence rate of $\mathrm{AE}$ in humans is increasing in Europe and Switzerland, and thus becoming an increasingly probable differential diagnosis.

\section{Statement of Ethics}

The authors have no ethical conflicts to disclose.

\section{Disclosure Statement}

The authors declare that they have no relevant financial interests.

\section{Funding Sources}

No external or internal funding was required for this case report.

\section{References}

1 Von der Weid NX: Spätfolgen nach Krebserkrankung und Krebstherapie. Pädiatrie 2005;3:24-27.

$12 \mathrm{Ng}$ AK, LaCasce A, Travis LB: Long-term complications of lymphoma and its treatment. J Clin Oncol 2011;29:1885-1892.

-3 Kebudi R, Özdemir GN: Secondary neoplasms in children treated for cancer. Curr Pediatr Rev 2017;13:34-41.

4 Martelli M, Ferreri AJ, Agostinelli C, Di Rocco A, Pfreundschuh M, Pileri SA: Diffuse large B-cell lymphoma. Crit Rev Oncol Hematol 2013;87:146-171.

5 National Guideline Alliance (UK): Non-Hodgkin's Lymphoma: Diagnosis and Management. London, National Institute for Health and Care Excellence (UK), 2016 (NICE Guideline, No 52).

-6 Mudie NY, Swerdlow AJ, Higgins CD, Smith P, Qiao Z, Hancock BW, Hoskin PJ, Linch DC: Risk of second malignancy after non-Hodgkin's lymphoma: a British Cohort Study. J Clin Oncol 2006;24:1568-1574.

-7 Tward JD, Wendland MM, Shrieve DC, et al: The risk of secondary malignancies over 30 years after the treatment of non-Hodgkin lymphoma. Cancer 2006;107:108-115.

-8 André M, Mounier N, Leleu X, Sonet A, Brice P, Henry-Amar M, Tilly H, Coiffier B, Bosly A, Morel P, et al: Second cancers and late toxicities after treatment of aggressive non-Hodgkin lymphoma with the ACVBP regimen: a GELA cohort study on 2,837 patients. Blood 2004;103:1222-1228.

9 Epidemiologie der Fuchsbandwurmerkrankungen in Deutschland - Daten des Echinokokkose Registers. Robert Koch Institut, Epidemiologisches Bulletin, April 2006 (Nr 15). 


\section{Case Reports in Oncology}

\begin{tabular}{l|l}
\hline Case Rep Oncol 2017;10:981-986 \\
\hline DOI: 10.1159/000481501 & $\begin{array}{l}\text { @ } 2017 \text { The Author(s). Published by S. Karger AG, Basel } \\
\text { www.karger.com/cro }\end{array}$ \\
\hline
\end{tabular}

Seidel et al.: A Rare Differential Diagnosis of an Adrenal Mass: A Case Report

10 Torgerson PR, Schweiger A, Deplazes P, et al: Alveolar echinococcosis: from a deadly disease to a wellcontrolled infection. Relative survival and economic analysis in Switzerland over the last 35 years. J Hepatol 2008;49:72-77.

-11 Brunetti E, Kern P, Vuitton DA; Writing Panel for the WHO-IWGE: Expert consensus for the diagnosis and treatment of cystic and alveolar echinococcosis in humans. Acta Trop 2010;114:1-16.

$\$ 12$ Huang M, Zheng H: Primary alveolar echinococcosis (Echinococcus multilocularis) of the adrenal gland: report of two cases. Int J Infect Dis 2013;17:e653-e655.

13 Spahn S, Helmchen B, Zingg U: Alveolar echinococcosis of the right adrenal gland: a case report and review of the literature. J Med Case Rep 2016;10:325.

14 Maru S, Yamashita N, Shinno Y: Adrenal multilocular echinococcosis: a case report. Nihon Hinyokika Gakkai Zasshi 2007;98:643-645.

15 Bulakçı M, Kartal MG, Yllmaz S, et al: Multimodality imaging in diagnosis and management of alveolar echinococcosis: an update. Diagn Interv Radiol 2016;22:247-256.

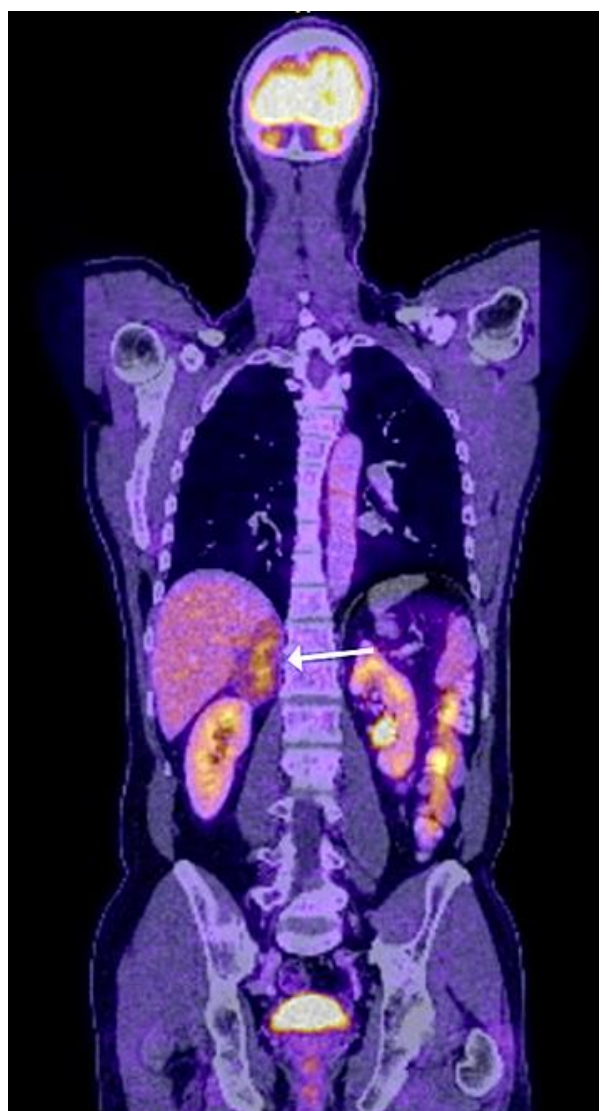

Fig. 1. PET CT scan showing the parasitic pseudotumorous lesion in the adrenal gland with elevated FDG uptake. 


\section{Case Reports in Oncology}
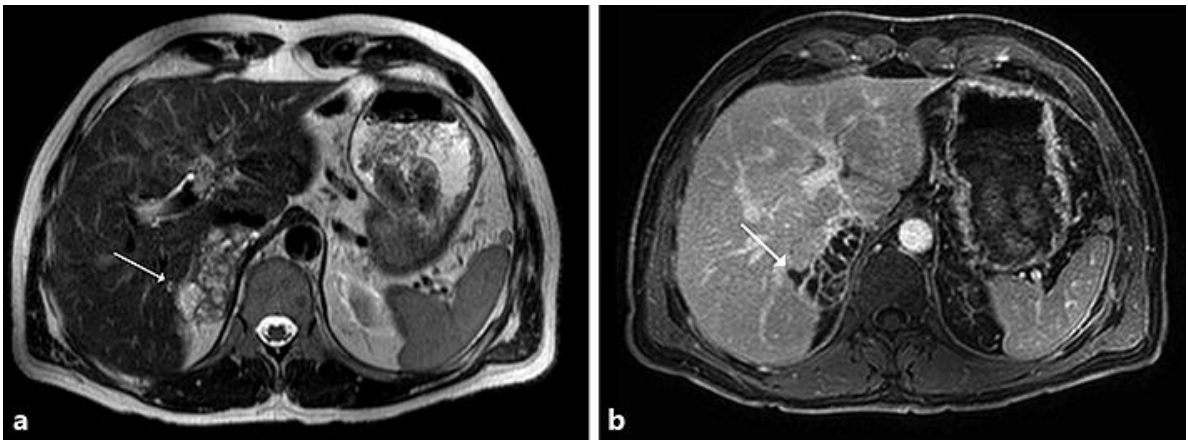

Fig. 2. MRI of the upper abdomen showing one parasitic lesion in liver segment 7 with infiltrative growth into the right adrenal gland. a T2 sequence. $\mathbf{b}$ T1 sequence.

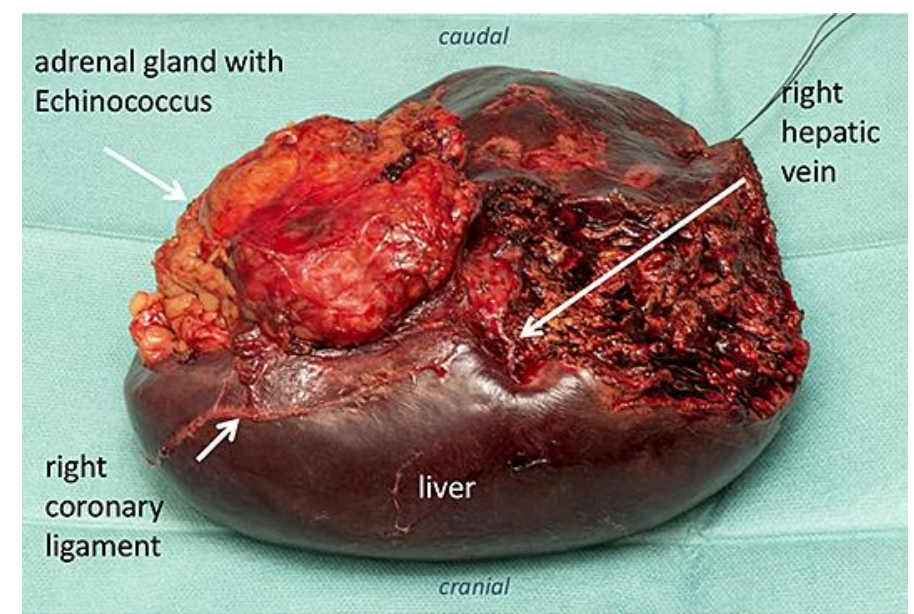

Fig. 3. Specimen after right hemihepatectomy (including segments 4, 5, 7, and 8) and right adrenalectomy. 